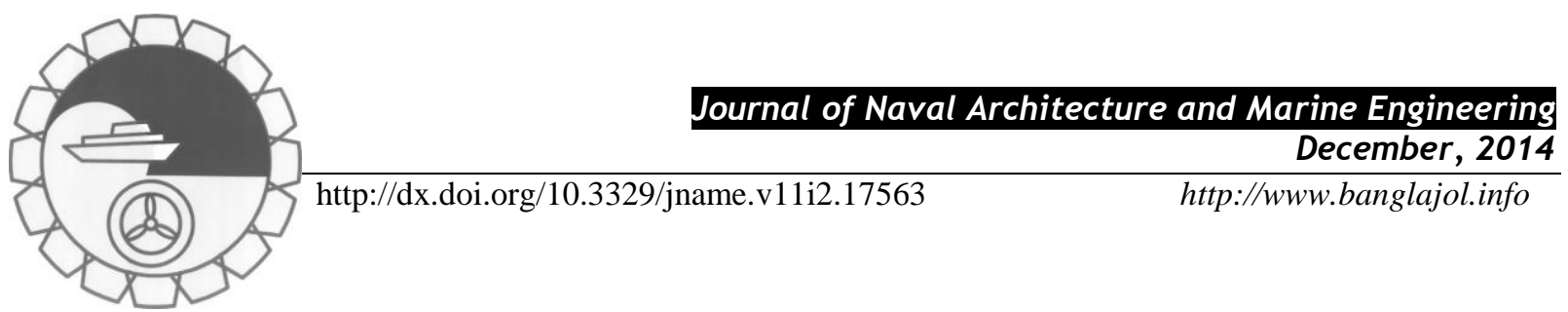

\title{
SORET EFFECTS DUE TO NATURAL CONVECTION IN A NON- NEWTONIAN FLUID FLOW IN POROUS MEDIUM WITH HEAT AND MASS TRANSFER
}

\author{
M.C.Raju $^{1 *}$ S.V.K.Varma ${ }^{2}$ \\ ${ }^{* 1}$ Department of Humanities and Sciences (Mathematics), Annamacharya Institute of Technology and Sciences, Rajampet - \\ 516126, A. P., INDIA, Email: mcrmaths@yahoo.co.in \\ ${ }^{2}$ Department of Mathematics, S. V. University, Tirupati - 517502, A.P., INDIA, Email: svijayakumarvarma@ yahoo.co.in
}

\begin{abstract}
:
The problem of unsteady MHD free convective, incompressible electrically conducting, nonNewtonian fluid through porous medium bounded by an infinite vertical porous plate in the presence of constant suction has been studied. A magnetic field of uniform strength is assumed to be applied normal to the plate. The equations governing the fluid flow which are highly nonlinear are reduced to linear by using perturbation method and have been solved subject to the relevant boundary conditions. It is noted that the velocity of the fluid is increased as Soret number and suction parameter increase, whereas reverse phenomenon is observed in case of magnetic field strength and sink strength.
\end{abstract}

Keywords: Non-Newtonian fluid; MHD; Free convection; heat and Mass transfer flow; and suction.

\section{Introduction}

Magneto hydrodynamics is currently undergoing a period of great enlargement and differentiation of subject matter. Free convection flow occurs frequently in nature, flows of fluid through porous media are of main interest now days and have attracted by many research scholars due to their applications in the science and technology. The study of hydro magnetic convective non-Newtonian fluid flows with heat and mass transfer in porous medium attracted many research due to its applications in many field like, soil physics, geophysics, aerodynamics and aeronautics. The interest in these new problems generates from their importance in liquid metals, electrolytes and ionized gases. Because of their varied importance, these flows have been studied by several authors-notable amongst them are Ferraro and Plumpton (1996) and Crammer and Pai (1973), Pal and Chatterjee (2010), Pal and Talkdar (2010), Chein (2004), Jha and Prasad (1991), Acharya et al. (2000), Kafousias and Raptis (1981), Kishore et al. (2010), Raju et al. (2008 \& 2011) . In all the above studies, the fluid models considered are Newtonian. But in view of the mechanism of non-Newtonian flows, which are used in various manufacturing processes, play a very important role in industrial applications and modern technology. Many authors like Choudary and Das (2013), Emmanuel and Khan (2006), Rafeal (2007), and Rushikumar and Sivaraj (2012), Hayat et al. (2010). Kelly et al. (1999), Reddy et al. (2010), investigated an unsteady free convective MHD non-Newtonian flow through a porous medium bounded by an infinite inclined porous plate. Motivated by the above cited work, in this paper, we consider an unsteady MHD free convective, thermal diffusive and viscos-elastic fluid with heat and mass transfer in porous medium bounded by an infinite vertical porous plate with heat absorption and viscous dissipation.

\section{Mathematical Formulation}

We consider a two-dimensional unsteady MHD free convection non-Newtonian mass transfer flow of an incompressible viscous fluid with and suction past an infinite vertical porous plate. In rectangular Cartesian coordinate system, we take $x^{l}$-axis along the infinite plate in the direction of flow and $y^{l}$-axis normal to it. All the fluid properties are constant except the density in the buoyancy force term. The Eckert number Ec and the magnetic Reynolds number $R m$ are small, so that the induced magnetic field can be neglected.

The governing equations for continuity, momentum, energy and concentration take the following form: 


$$
\begin{aligned}
& \frac{\partial v^{1}}{\partial y^{1}}=0 \\
& \frac{\partial u^{1}}{\partial t^{1}}+v^{1} \frac{\partial u^{1}}{\partial y^{1}}=g \beta\left(T^{1}-T_{\infty}\right)+g \beta^{1}\left(C^{1}-C_{\infty}\right)+v \frac{\partial^{2} u^{1}}{\partial y^{1^{2}}}+B_{1}\left(\frac{\partial^{3} u^{1}}{\partial t^{1} \partial y^{1^{2}}}+v^{1} \frac{\partial^{3} u^{1}}{\partial y^{1^{3}}}\right)-\frac{\sigma B_{0}^{2}}{\rho} u^{1}-\frac{v}{k} u^{1} \\
& \frac{\partial T^{1}}{\partial t^{1}}+v^{1} \frac{\partial T^{1}}{\partial y^{1}}=\frac{K_{T}}{\rho C_{p}} \frac{\partial^{2} T^{1}}{\partial y^{1^{2}}}+A^{1}\left(T^{1}-T_{\infty}\right)+\frac{v}{C_{p}}\left(\frac{\partial u^{1}}{\partial y^{1}}\right)^{2} \\
& \frac{\partial C^{1}}{\partial t^{1}}+v^{1} \frac{\partial C^{1}}{\partial y^{1}}=D \frac{\partial^{2} C^{1}}{\partial y^{1^{2}}}+D_{1} \frac{\partial^{2} T^{1}}{\partial y^{1^{2}}}
\end{aligned}
$$

The boundary conditions relevant to the problem are;

$$
\begin{aligned}
& u^{1}=0, v^{1}=-v_{0}, T^{1}=T_{w}+\varepsilon\left(T_{w}-T_{\infty}\right) e^{i \omega t}, C^{1}=C_{w}+\varepsilon\left(C_{w}-C_{\infty}\right) e^{i \omega t} \text { at } y^{1}=0 \\
& u^{1} \rightarrow 0, \quad T^{1} \rightarrow T_{\infty}, \quad C^{1} \rightarrow C_{\infty} \quad \text { as } y^{1} \rightarrow \infty
\end{aligned}
$$

Where $\mathrm{u}^{1}$ the dimensional velocity component, $\mathrm{T}^{1}$ the dimensional temperature distribution, $\mathrm{C}^{1}$ the dimensional concentration distribution, $T_{\infty}^{1}$ dimensional temperature far away from the plate, $C_{\infty}^{1}$ dimensional concentration far away from the plate, $T_{w}$ temperature near the plate, $C_{w}$ concentration near the plate, $t^{l}$ dimensional time, $x^{l}$, $y^{l}$ dimensional Cartesian coordinates along and normal to the plate, $B_{0}$ transverse magnetic field, $g$ is the acceleration due to gravity, $v^{l}$ dimensional suction velocity, $B_{l}$ kinematical non-Newtonian parameter, $k$ is the permeability of the porous medium, $\beta$ volumetric expansion coefficient, $\beta^{l}$ volumetric expansion coefficient of mass diffusion, $\vartheta$ kinematic viscosity, $\rho$ is the density of the fluid, $\sigma$ electrical conductivity, $K_{T}$ thermal conductivity, $\varepsilon$ is a perturbation parameter $(<<1), c_{p}$ is specific heat, $A^{l}$ is heat absorption coefficient, $\omega$ is frequency parameter, $D$ is mass diffusivity, and $D_{l}$ thermal diffusivity.

On introducing the following non-dimensional quantities,

$$
\begin{aligned}
& y=\frac{y^{1} v_{0}}{v}, u=\frac{u^{1}}{v_{0}}, t=\frac{t^{1} v_{0}^{2}}{4 v}, T=\frac{T^{1}-T_{\infty}}{T_{w}-T_{\infty}}, v=\frac{\mu}{\rho}, A=\frac{A^{1} v_{0}^{2}}{4 v}, R_{m}=\frac{B_{1} v_{0}^{2}}{v^{2}}, C=\frac{C^{1}-C_{\infty}}{C_{w}-C_{\infty}}, G_{r}=\frac{g \beta v\left(T_{w}-T_{\infty}\right)}{v_{0}{ }^{3}}, \\
& G_{m}=\frac{g \beta^{1} v\left(C_{w}-C_{\infty}\right)}{v_{0}^{3}}, M=\frac{\sigma v B_{0}^{2}}{\rho v_{0}^{2}}, K=\frac{v^{2} k}{v_{0}^{2}}, P_{r}=\frac{v \rho C_{p}}{K_{T}}, E_{C}=\frac{v_{O}^{2}}{C_{p}\left(T_{w}-T_{\infty}\right)}, \\
& S_{C}=\frac{v}{D}, S_{\mathrm{O}}=\frac{D_{1}}{v}\left(\frac{T_{w}-T_{\infty}}{C_{w}-C_{\infty}}\right)
\end{aligned}
$$

in set of Equations (2)- (4), we obtain the governing equations in the dimensionless form as

$$
\begin{aligned}
& \frac{1}{4} \frac{\partial u}{\partial t}-\frac{\partial u}{\partial y}=G_{r} T+G_{m} C+\frac{\partial^{2} u}{\partial y^{2}}+R_{m}\left(\frac{1}{4} \frac{\partial^{3} u}{\partial t \partial y^{2}}-\frac{\partial^{3} u}{\partial y^{3}}\right)-M_{1} u \\
& \frac{P_{r}}{4} \frac{\partial T}{\partial t}-P_{r} \frac{\partial T}{\partial y}=\frac{\partial^{2} T}{\partial y^{2}}+\frac{A P_{r}}{4} T+P_{r} E_{c}\left(\frac{\partial u}{\partial y}\right)^{2} \\
& \frac{1}{4} \frac{\partial C}{\partial t}-\frac{\partial C}{\partial y}=\frac{1}{S_{c}} \frac{\partial^{2} C}{\partial y^{2}}+S_{0}\left(\frac{\partial^{2} T}{\partial y^{2}}\right)
\end{aligned}
$$

$M_{1}=M+1 / K, G r$ is Grashof's number, $G m$ is modified Grashof's number, $R_{m}$ is dimensionless noNewtonian parameter, $M$ the magnetic parameter, and $K$ is permeability parameter, $\operatorname{Pr}$ Prandtl number, $E c$ is Eckert number, $S c$ Schmidt number, and $S_{0}$ is Soret number. Now the boundary conditions (4) reduce into the following form

$$
\begin{array}{lllll}
u=0, & T=1+\varepsilon e^{i \omega t}, & C=1+\varepsilon e^{i \omega t} & \text { at } & y=0 \\
u \rightarrow 0, & T \rightarrow 0, & C \rightarrow 0 & \text { as } & y \rightarrow \infty
\end{array}
$$

\section{Solution of the Problem}

To solve the partial differential equations (6), (7), (8) subject to the boundary conditions (9), the velocity $(u)$, the temperature $(T)$ and the concentration $(C)$ in the neighborhood of the plate are assumed to be of the form ; 


$$
\begin{aligned}
& u(y, t)=f_{0}(y)+\varepsilon e^{i \omega t} f_{1}(y)=f_{0}+\varepsilon e^{i \omega t} f_{1} \\
& T(y, t)=g_{0}(y)+\varepsilon e^{i \omega t} g_{1}(y)=g_{0}+\varepsilon e^{i \omega t} g_{1} \\
& C(y, t)=h_{0}(y)+\varepsilon e^{i \omega t} h_{1}(y)=h_{0}+\varepsilon e^{i \omega t} h_{1}
\end{aligned}
$$

Substituting Equations (11), (12), (13) in Equations (7), (8), (9) and equating the coefficientsof $\varepsilon^{0}, \varepsilon^{1}$ (neglecting $\varepsilon^{2}$ terms etc.,), we obtain the following set of ordinary differential equations.

$$
\begin{aligned}
& R_{m} f_{0}{ }^{111}-f_{0}{ }^{11}-f_{0}{ }^{1}+M_{1} f_{0}=G_{r} g_{0}+G_{m} h_{0} \\
& R_{m} f_{1}{ }^{11}-\left(1+\frac{R_{m} i \omega}{4}\right) f_{1}{ }^{11}-f_{1}{ }^{1}+\left(M_{1}+\frac{i \omega}{4}\right) f_{1}=G_{r} g_{1}+G_{m} h_{1} \\
& g_{0}{ }^{11}+P_{r} g_{0}{ }^{1}+\frac{A P_{r}}{4} g_{0}=-P_{r} E_{c}\left(f_{0}{ }^{1}\right)^{2} \\
& g_{1}{ }^{11}+P_{r} g_{1}{ }^{1}+\frac{P_{r}}{4}(A-i \omega) g_{1}=-2 P_{r} E_{c} f_{0}{ }^{1} f_{1}{ }^{1} \\
& h_{0}{ }^{11}+S_{c} h_{0}{ }^{1}=-S_{0} S_{c} g_{0}{ }^{11} \\
& h_{1}{ }^{11}+S_{c} h_{1}{ }^{1}-\frac{S_{c} i \omega}{4} h_{1}=-S_{0} S_{c} g_{1}{ }^{11}
\end{aligned}
$$

And the corresponding boundary conditions are

$$
\begin{aligned}
& u_{0}=u_{1}=0, \quad T_{0}=T_{1}=1, \quad C_{0}=C_{1}=1 \quad \text { at } \quad y=0 \\
& u_{0}, u_{1} \rightarrow 0, \quad T_{0}, T_{1} \rightarrow 0, \quad C_{0}, C_{1} \rightarrow 0 \quad \text { as } \quad y \rightarrow \infty
\end{aligned}
$$

In Equations (14) and (15), due to presence of elasticity, we get $3^{\text {rd }}$ order differential equations. To solve these differential equations we need three boundary conditions, but we have only two. So we assume the solutions as;

$$
\begin{array}{lll}
f_{0}=f_{00}+R_{m} f_{01} & g_{0}=g_{00}+R_{m} g_{01} & h_{0}=h_{00}+R_{m} h_{01} \\
f_{1}=f_{10}+R_{m} f_{11} & g_{1}=g_{10}+R_{m} g_{11} & h_{1}=h_{10}+R_{m} h_{11}
\end{array}
$$

Substitute Equations (21) in the Equations (14), (15), (16), (17), (18), (19), equating the coefficients of $R_{m}{ }^{0}$, $R_{m}{ }^{1}$ (neglecting higher order terms), we obtain the following set of ordinary differential equations.

\section{Zero order:}

$$
\begin{aligned}
& f_{00}{ }^{11}+f_{00}{ }^{1}-M_{1} f_{00}=-G_{r} g_{00}-G_{m} h_{00} \\
& f_{10}{ }^{11}+f_{10}{ }^{1}-\left(M_{1}+\frac{i \omega}{4}\right) f_{10}=-G_{r} g_{10}-G_{m} h_{10} \\
& g_{00}{ }^{11}+P_{r} g_{00}{ }^{1}+\frac{A P_{r}}{4} g_{00}=-P_{r} E_{c}\left(f_{00}{ }^{1}\right)^{2} \\
& g_{10}{ }^{11}+P_{r} g_{10}{ }^{1}+\frac{P_{r}}{4}(A-i \omega) g_{10}=-P_{r} E_{c} f_{00}{ }^{1} f_{10}{ }^{1} \\
& h_{00}{ }^{11}+S_{c} h_{00}{ }^{1}=-S_{0} S_{c} g_{00}{ }^{11} \\
& h_{10}{ }^{11}+S_{c} h_{10}{ }^{1}-\frac{S_{c} i \omega}{4} h_{10}=-S_{0} S_{c} g_{10}{ }^{11}
\end{aligned}
$$

First order:

$$
\begin{aligned}
& f_{01}{ }^{11}+f_{01}{ }^{1}-M_{1} f_{01}=f_{00}{ }^{111}-G_{r} g_{01}-G_{m} h_{01} \\
& f_{11}{ }^{11}+f_{11}{ }^{1}-\left(M_{1}+\frac{i \omega}{4}\right) f_{11}=f_{10}{ }^{111}-\frac{i \omega}{4} f_{10}{ }^{11}-G_{r} g_{11}-G_{m} h_{11} \\
& g_{01}{ }^{11}+P_{r} g_{01}{ }^{1}+\frac{A P_{r}}{4} g_{01}=-2 P_{r} E_{c} f_{00}{ }^{1} f_{01}{ }^{1}
\end{aligned}
$$




$$
\begin{aligned}
& g_{11}{ }^{11}+P_{r} g_{11}{ }^{1}+\frac{P_{r}}{4}(A-i \omega) g_{11}=-2 P_{r} E_{c}\left(f_{00}{ }^{1} f_{11}{ }^{1}+f_{01}{ }^{1} f_{10}{ }^{1}\right) \\
& h_{01}{ }^{11}+S_{c} h_{01}{ }^{1}=-S_{0} S_{c} g_{01}{ }^{11} \\
& h_{11}{ }^{11}+S_{c} h_{11}{ }^{1}-\frac{S_{c} i \omega}{4} h_{11}=-S_{0} S_{c} g_{11}{ }^{11}
\end{aligned}
$$

In order to solve the above non-linear system of equations (22) to (33), expand $f_{00}, f_{01}, f_{10}, f_{11}, g_{00}, g_{01}, g_{10}$, $\mathrm{g}_{11}, \mathrm{~h}_{00}, \mathrm{~h}_{01}, \mathrm{~h}_{10}, \mathrm{~h}_{11}$ in powers of Eckert number $\left(\mathrm{E}_{\mathrm{c}}<<<1\right)$ for all incompressible fluids. So, we assume that

$$
\begin{array}{ccc}
f_{00}=f_{000}+E_{c} f_{001}+o\left(E_{c}{ }^{2}\right) & g_{00}=g_{000}+E_{c} g_{001}+o\left(E_{c}{ }^{2}\right) & h_{00}=h_{000}+E_{c} h_{001}+o\left(E_{c}{ }^{2}\right) \\
f_{01}=f_{010}+E_{c} f_{011}+o\left(E_{c}{ }^{2}\right) & g_{01}=g_{010}+E_{c} g_{011}+o\left(E_{c}{ }^{2}\right) & h_{01}=h_{010}+E_{c} h_{011}+o\left(E_{c}{ }^{2}\right) \\
f_{10}=f_{100}+E_{c} f_{101}+o\left(E_{c}{ }^{2}\right) & g_{10}=g_{100}+E_{c} g_{101}+o\left(E_{c}{ }^{2}\right) & h_{10}=h_{100}+E_{c} h_{101}+o\left(E_{c}{ }^{2}\right) \\
f_{11}=f_{110}+E_{c} f_{111}+o\left(E_{c}{ }^{2}\right) & g_{11}=g_{110}+E_{c} g_{111}+o\left(E_{c}{ }^{2}\right) & h_{11}=h_{110}+E_{c} h_{111}+o\left(E_{c}{ }^{2}\right)
\end{array}
$$

Using Equations (34) in Equations (22) to (33) and equating the coefficients of $\mathrm{E}_{\mathrm{c}}{ }^{0}, \mathrm{E}_{\mathrm{c}}{ }^{1}$ (neglecting the coefficients of $\mathrm{E}_{\mathrm{c}}{ }^{2}$ etc.,), we get the following set of differential equations;

\section{Zero order terms:}

$$
\begin{aligned}
& f_{000}{ }^{11}+f_{000}{ }^{1}-M_{1} f_{000}=-G_{r} g_{000}-G_{m} h_{000} \\
& f_{100}{ }^{11}+f_{100}{ }^{1}-\left(M_{1}+\frac{i \omega}{4}\right) f_{100}=-G_{r} g_{100}-G_{m} h_{100} \\
& g_{000}{ }^{11}+P_{r} g_{000}{ }^{1}+\frac{A P_{r}}{4} g_{000}=0 \\
& g_{100}{ }^{11}+P_{r} g_{100}{ }^{1}+\frac{P_{r}}{4}(A-i \omega) g_{100}=0 \\
& h_{000}{ }^{11}+S_{c} h_{000}{ }^{1}=-S_{0} S_{c} g_{000}{ }^{11} \\
& h_{100}{ }^{11}+S_{c} h_{100}{ }^{1}-\frac{S_{c} i \omega}{4} h_{100}=-S_{0} S_{c} g_{100}{ }^{11} \\
& f_{010}{ }^{11}+f_{010}{ }^{1}-M_{1} f_{010}=f_{000}{ }^{111}-G_{r} g_{010}-G_{m} h_{010} \\
& f_{110}{ }^{11}+f_{110}{ }^{1}-\left(M_{1}+\frac{i \omega}{4}\right) f_{110}=f_{100}{ }^{111}-\frac{i \omega}{4} f_{100}{ }^{11}-G_{r} g_{110}-G_{m} h_{110} \\
& g_{010}{ }^{11}+P_{r} g_{010}{ }^{1}+\frac{A P_{r}}{4} g_{010}=0 \\
& g_{110}{ }^{11}+P_{r} g_{110}{ }^{1}+\frac{P_{r}}{4}(A-i \omega) g_{110}=0 \\
& h_{010}{ }^{11}+S_{c} h_{010}{ }^{1}=-S_{0} S_{c} g_{010}{ }^{11} \\
& h_{110}{ }^{11}+S_{c} h_{110}{ }^{1}-\frac{S_{c} i \omega}{4} h_{110}=-S_{0} S_{c} g_{110}{ }^{11}
\end{aligned}
$$

First order terms: 


$$
\begin{aligned}
& f_{101}{ }^{11}+f_{101}{ }^{1}-\left(M_{1}+\frac{i \omega}{4}\right) f_{101}=-G_{r} g_{101}-G_{m} h_{101} \\
& g_{001}{ }^{11}+P_{r} g_{001}{ }^{1}+\frac{A P_{r}}{4} g_{001}=-P_{r} f_{000}^{1}{ }^{2} \\
& g_{101}{ }^{11}+P_{r} g_{101}{ }^{1}+\frac{P_{r}}{4}(A-i \omega) g_{101}=-P_{r} f_{000}{ }^{1} f_{100}{ }^{1} \\
& h_{001}{ }^{11}+S_{c} h_{001}{ }^{1}=-S_{0} S_{c} g_{001}{ }^{11} \\
& h_{101}{ }^{11}+S_{c} h_{101}{ }^{1}-\frac{S_{c} i \omega}{4} h_{101}=-S_{0} S_{c} g_{101}{ }^{11} \\
& f_{011}{ }^{11}+f_{011}{ }^{1}-M_{1} f_{011}=f_{001}{ }^{111}-G_{r} g_{011}-G_{m} h_{011} \\
& f_{111}{ }^{11}+f_{111}{ }^{1}-\left(M_{1}+\frac{i \omega}{4}\right) f_{111}=f_{101}{ }^{111}-\frac{i \omega}{4} f_{101}{ }^{11}-G_{r} g_{111}-G_{m} h_{111} \\
& g_{011}{ }^{11}+P_{r} g_{011}{ }^{1}+\frac{A P_{r}}{4} g_{011}=-2 P_{r} f_{000}{ }^{1} f_{010}{ }^{1} \\
& g_{111}{ }^{11}+P_{r} g_{111}{ }^{1}+\frac{P_{r}}{4}(A-i \omega) g_{111}=-2 P_{r}\left(f_{000}{ }^{1} f_{110}{ }^{1}+f_{010}{ }^{1} f_{100}{ }^{1}\right) \\
& h_{011}{ }^{11}+S_{c} h_{011}{ }^{1}=-S_{0} S_{c} g_{011}{ }^{11} \\
& h_{111}{ }^{11}+S_{c} h_{111}{ }^{1}-\frac{S_{c} i \omega}{4} h_{111}=-S_{0} S_{c} g_{111}{ }^{11}
\end{aligned}
$$

And the boundary conditions (20) reduce to

$$
\begin{aligned}
& f_{000}=f_{010}=f_{001}=f_{011}=f_{100}=f_{110}=f_{101}=f_{111}=0 \\
& g_{000}=1, g_{010}=g_{001}=g_{011}=0, g_{100}=1, g_{110}=g_{101}=g_{111}=0 \\
& h_{000}=1, h_{010}=h_{001}=h_{011}=0, h_{100}=1, h_{110}=h_{101}=h_{111}=0 \\
& f_{000} \rightarrow f_{010} \rightarrow f_{001} \rightarrow f_{011} \rightarrow f_{100} \rightarrow f_{110} \rightarrow f_{101} \rightarrow f_{111} \rightarrow 0 \\
& g_{000} \rightarrow g_{010} \rightarrow g_{001} \rightarrow g_{011} \rightarrow g_{100} \rightarrow g_{110} \rightarrow g_{101} \rightarrow g_{111} \rightarrow 0 \quad \text { As } y \rightarrow \infty \\
& h_{000} \rightarrow h_{010} \rightarrow h_{001} \rightarrow h_{011} \rightarrow h_{100} \rightarrow h_{110} \rightarrow h_{101} \rightarrow h_{111} \rightarrow 0 \\
& \text { At } \mathrm{y}=0
\end{aligned}
$$

Solving these differential equations (35) to (58), using the above boundary conditions, also making use of Equations (34) and the approximate substitutions in Equations (21), we obtain velocity $(u)$, temperature $(T)$ and concentration $(C)$.

\section{Skin-Friction:}

The expression for the skin-friction $(\tau)$ at the plate is,

$$
\tau=\left(\frac{d u}{d y}\right)_{y=0}=\left(\frac{d f_{0}}{d y}\right)_{y=0}+\varepsilon e^{i \omega t}\left(\frac{d f_{1}}{d y}\right)_{y=0}=\quad A_{165}+\varepsilon e^{i \omega t} A_{166}
$$

\section{Rate of heat transfer:}

The expression for the rate of heat transfer in terms of Nusselt number $\left(\mathrm{N}_{\mathrm{u}}\right)$ is, 


$$
N_{u}=\left(\frac{d T}{d y}\right)_{y=0}=\left(\frac{d g_{0}}{d y}\right)_{y=0}+\varepsilon e^{i \omega t}\left(\frac{d g_{1}}{d y}\right)_{y=0}=A_{167}+\varepsilon e^{i \omega t} A_{168}
$$

\section{Rate of mass transfer:}

The expression for the rate of mass transfer in the form of Sherwood number $\left(\mathrm{S}_{\mathrm{h}}\right)$ is,

$$
S_{h}=\left(\frac{d C}{d y}\right)_{y=0}=\left(\frac{d h_{0}}{d y}\right)_{y=0}+\varepsilon e^{i \omega t}\left(\frac{d h_{1}}{d y}\right)_{y=0}=A_{169}+\varepsilon e^{i \omega t} A_{170}
$$

\section{Results and Discussion}

In order to get physical insight into the problem, the velocity, the temperature, the concentration, skin friction, rate of heat transfer and rate of mass transfer have been discussed by assigning numerical values for $\mathrm{M}, \mathrm{Pr}$, and A while keeping $\mathrm{Rm}=0.05, \mathrm{w}=5.0, \mathrm{e}=2.0, \mathrm{Ec}=0.001$. Velocity profiles are displayed in Figs. 1-3, with the variations in the physical parameters like magnetic parameter M, sink parameter A, and Soret number So. We have choose $\operatorname{Pr}=0.025$ and 1.0 which represent mercury and electrolytic solution respectively. For the validity of our results, we compared our results with the data sets of Reddy et al. [15] at the absence of mass transfer. From this it is noticed that our results are in good agreement with the existing results (see Fig. 9). In Fig. 1 the effects of magnetic parameter $\mathrm{M}$ on velocity is presented. From this figure it is noticed that velocity decreases as magnetic parameter M increases. So it indicates that external magnetic field that acts as Lorentz's force which suppresses the free convection. The effect of thermal diffusion on velocity is presented in Fig. 2, it is noticed that velocity increases as Soret number So increases as expected. In Fig. 3 velocity profiles are presented with the variations in sink parameter A, we observe that velocity increases as sink parameter A increases. In the Figs. 4-7, variations in temperature distribution are presented for various values of sink parameter A, soret number So, Schmidt number Sc and magnetic parameter M. From these figures it is interesting to notice that, temperature decreases with the increase in suction parameter A, soret number So, Schmidt number Sc respectively, where as it shows reverse action in the case of magnetic parameter M. Similarly, the effects of soret number So on concentration are presented in Fig. 8, the concentration decreases as soret number So increases. Therefore, we have noticed that Velocity and Temperature are greater for mercury than that of electrolytic solution. From Table 1, it can be observed that skin friction increases with an increase in Hartmann number where as it shows reverse effect in the case of Grashof's number, modified Grashof's number, dimensionless non Newtonian parameter and permeability parameter. From Table 2, it is noticed that rate of heat transfer decreases with an increase in the sink strength. From Table 3, we see that rate of mass transfer also decreases with an increase in thermal diffusion number.

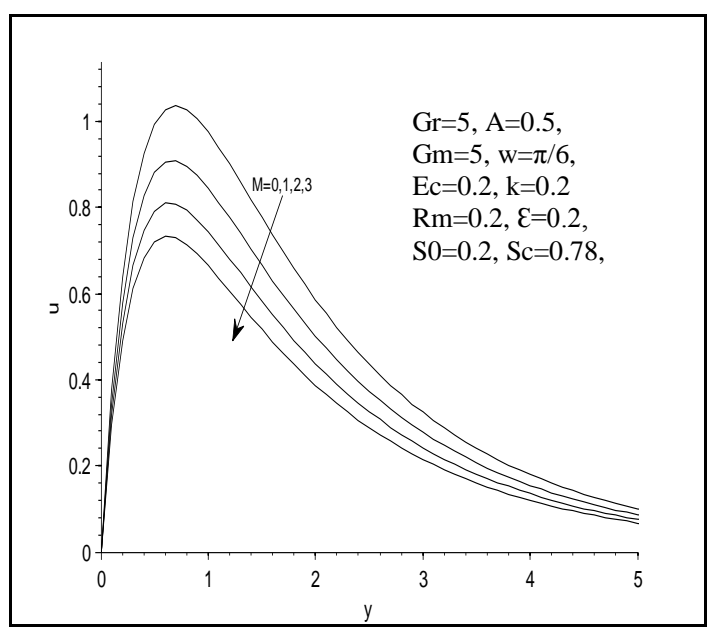

Fig. 1: Effects of magnetic parameter $\mathrm{M}$ on velocity

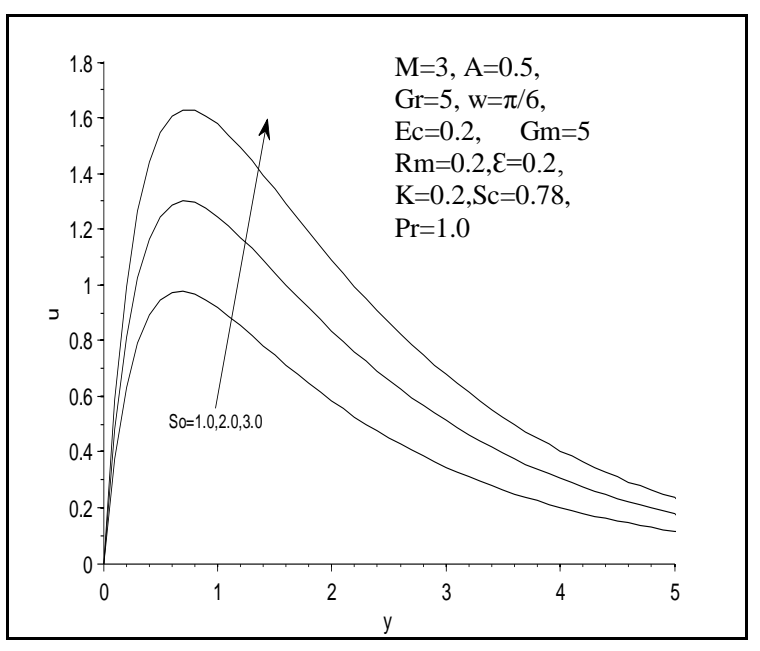

Fig. 2: Effects of Soret number on velocity 


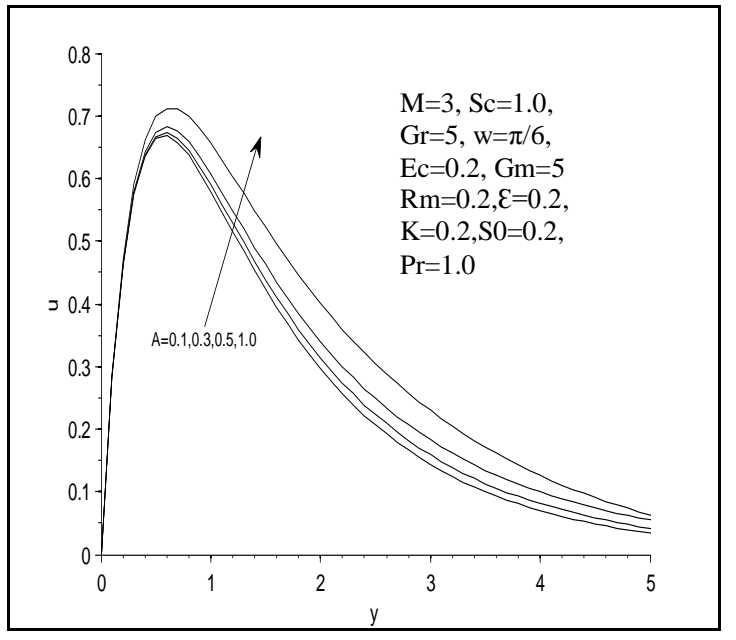

Fig. 3: Effects of Suction parameter on velocity

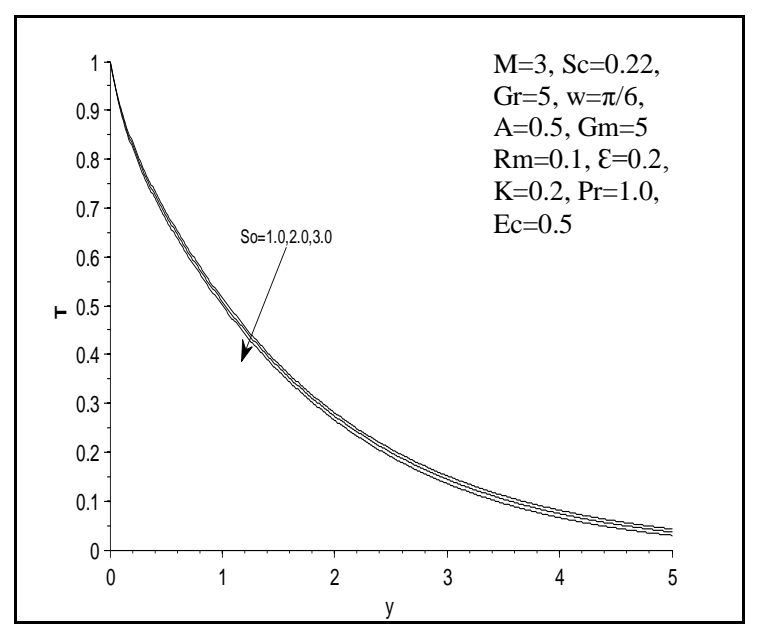

Fig. 5: Effects of Soret number on Temperature

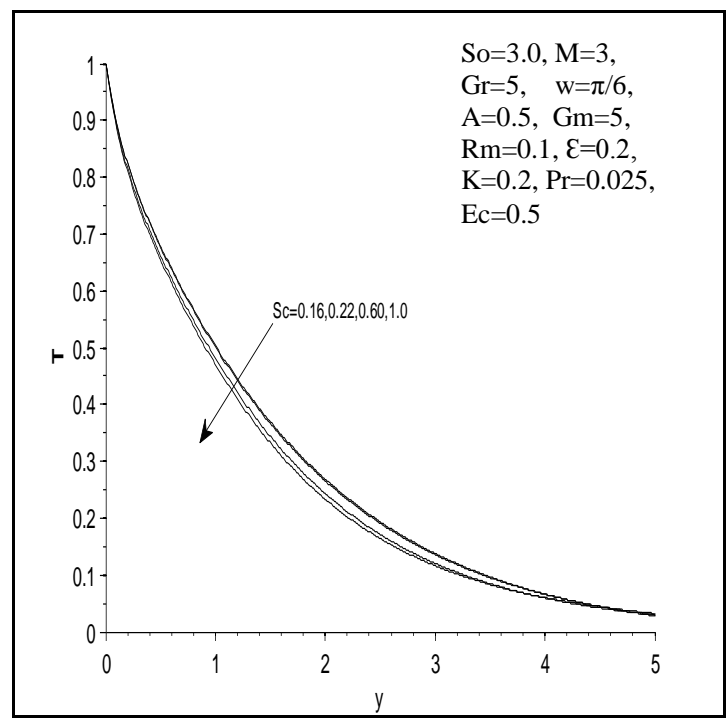

Fig. 7: Effects of Schmidt number on Temperature

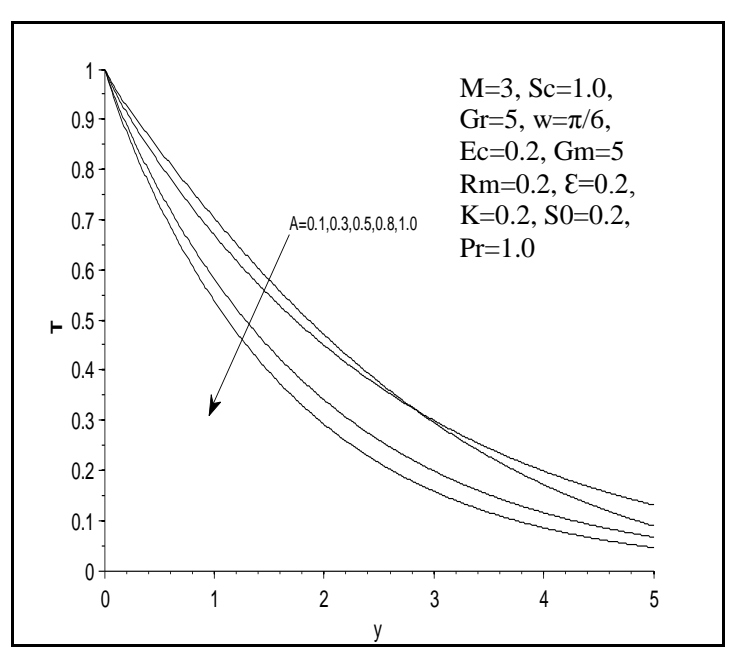

Fig. 4: Effects of Suction parameter on Temperature

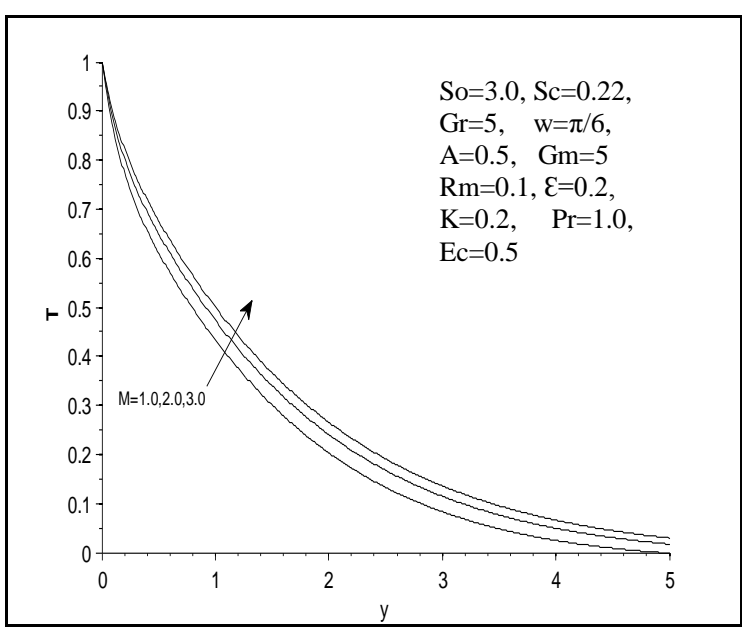

Fig. 6: Effects of $\mathrm{M}$ on Temperature

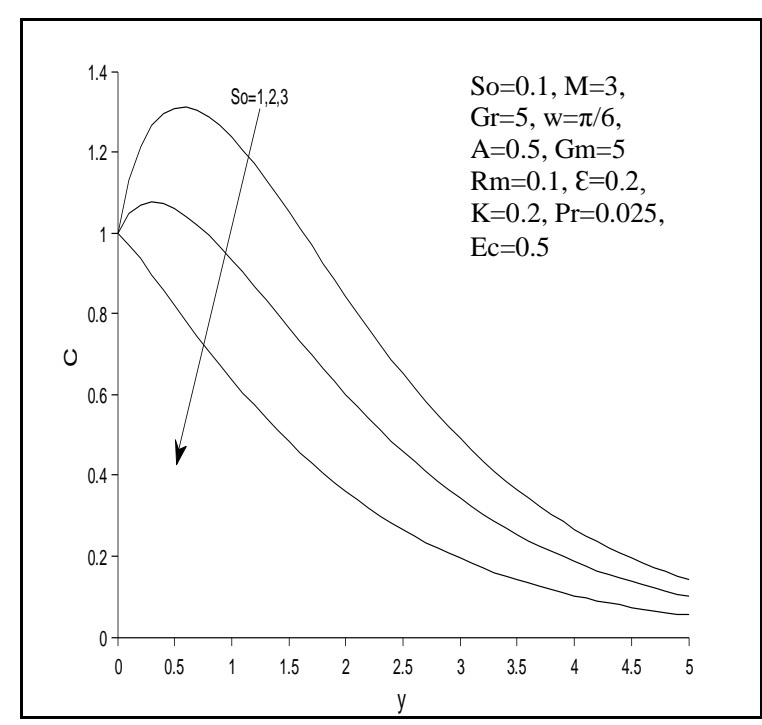

Fig. 8: Effects of Soret number on Concentration 


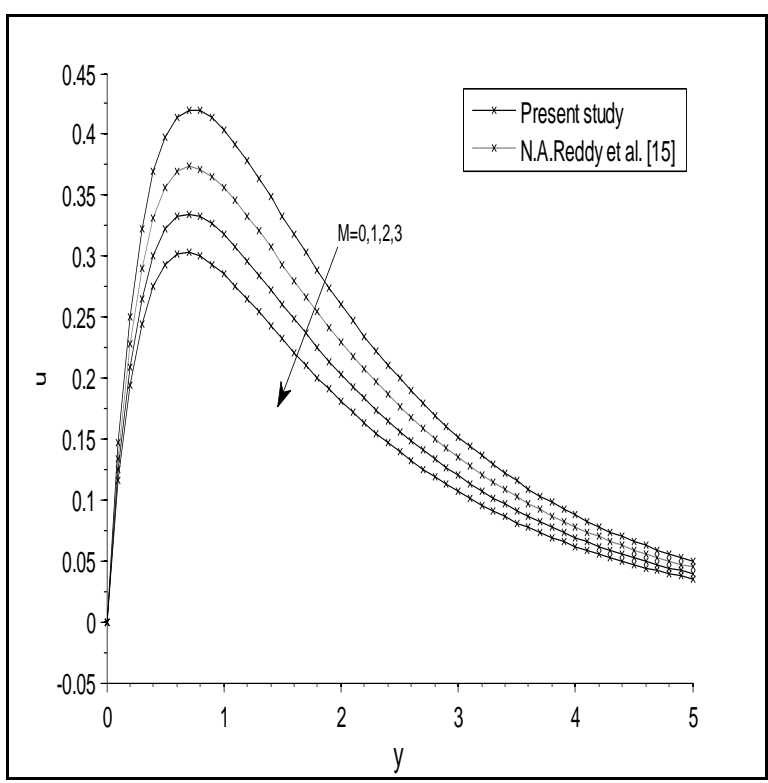

Table 1: Variations in rate of heat transfer

\begin{tabular}{|l|l|l|l|}
\hline Pr & Ec & A & Nu \\
\hline $\mathbf{0 . 7 1}$ & 0.1 & 0.1 & -2.6694 \\
\hline $\mathbf{0 . 7 4}$ & 0.1 & 0.1 & -3.0096 \\
\hline $\mathbf{0 . 7 7}$ & 0.1 & 0.1 & -3.4449 \\
\hline $\mathbf{0 . 7 1}$ & 0.2 & 0.1 & -1.0678 \\
\hline $\mathbf{0 . 7 1}$ & 0.3 & 0.1 & -2.4025 \\
\hline $\mathbf{0 . 7 1}$ & 0.1 & 0.2 & -3.3648 \\
\hline $\mathbf{0 . 7 1}$ & 0.1 & 0.3 & -3.9998 \\
\hline
\end{tabular}

Fig. 9: Effects of $M$ on velocity at the absence of $S c$ and $S O$

\section{Conclusion}

In this paper, the effect of unsteady free convective MHD non-Newtonian flow through a porous medium with suction bounded by an infinite porous plate has been studied numerically. Neglecting the induced magnetic field, the equations governing the velocity, the temperature and the concentration of the fluid are solved by multi-parameter perturbation technique in terms of dimensionless parameters. The conclusions are summarized as follows: External magnetic field suppresses the free convection flow. The velocity for viscous fluid is more than that of viscos-elastic fluids. The temperature of the fluid is more for mercury than for an electrolytic solution. Skin friction increases with an increase in permeability of porous medium and Hartmann number. Rate of heat transfer decreases with an increase in the sink strength and rate of mass transfer also decreases with an increase in thermal diffusion number.

Table 2: Variations in Skin-friction

\begin{tabular}{|l|l|l|l|l|l|}
\hline Gr & Gm & Rm & K & M & $\tau$ \\
\hline $\mathbf{5}$ & 5 & 0.1 & 0.6 & 2 & -2.9074 \\
\hline $\mathbf{1 0}$ & 5 & 0.1 & 0.6 & 2 & -5.1735 \\
\hline $\mathbf{1 5}$ & 5 & 0.1 & 0.6 & 2 & -7.7128 \\
\hline $\mathbf{5}$ & 10 & 0.1 & 0.6 & 2 & -1.5082 \\
\hline $\mathbf{5}$ & 15 & 0.1 & 0.6 & 2 & -4.2167 \\
\hline $\mathbf{5}$ & 5 & 0.2 & 0.6 & 2 & -5.8149 \\
\hline $\mathbf{5}$ & 5 & 0.3 & 0.6 & 2 & -8.7223 \\
\hline $\mathbf{5}$ & 5 & 0.1 & 0.7 & 2 & -2.8623 \\
\hline $\mathbf{5}$ & 5 & 0.1 & 0.8 & 2 & -1.1020 \\
\hline $\mathbf{5}$ & 5 & 0.1 & 0.6 & 3 & -3.0838 \\
\hline $\mathbf{5}$ & 5 & 0.1 & 0.6 & 4 & -2.6694 \\
\hline
\end{tabular}

Table 3: Variations in rate of mass transfer

\begin{tabular}{|l|l|l|}
\hline Se & So & Sh \\
\hline $\mathbf{0 . 2 2}$ & 4 & -3.5529 \\
\hline $\mathbf{0 . 2 6}$ & 4 & -3.8977 \\
\hline $\mathbf{0 . 3 0}$ & 4 & -4.2597 \\
\hline $\mathbf{0 . 2 2}$ & 5 & -4.0815 \\
\hline $\mathbf{0 . 2 2}$ & 6 & -4.6108 \\
\hline
\end{tabular}




\section{Reference}

Acharya, M., Dash, G.C. and Singh, L.P. (2000): Magnetic Field Effects On The Free Convection And Mass Transfer Flow Through Porous Medium With Constant Suction And Constant Heat Flux, Indian Journal of pure and applied mathematics., Vol. 31, No.1, pp.1-18.

Chen, C. H. (2004): Combined Heat and Mass Transfer in MHD Free Convection from a Vertical Wavy Surface, International journal of Engineering Science, Vol. 42, pp. 699-713. http://dx.doi.org/10.1016/j.ijengsci.2003.09.002

Choudhury, R. and Das, S. K. (2013): Mixed Convective No-Newtonian MHD Flow With Ohmic Heating. International Journal of computer applications, Vol. 68, No. 10, 7-13.

Crammer, K.P. and Pai, S.L. (1973): Magneto-Fluid Dynamics For Engineers And Applied Physicist, Mc-Graw Hill book co., New york.

Emmanuel, S. and Khan, S.K. (2006): On Heat And Mass Transfer In A Viscoelastic Boundary Layer Flow Over An Exponentially Stretching Sheet. International Journal of Thermal Sciences, Vol. 45, pp. 819-828.

http://dx.doi.org/10.1016/j.ijthermalsci.2005.11.002

Ferraro V. C. A. and Plumption. C. (1966): An Introduction To Magneto Fluid Mechanics, Clarandon Press, Oxford.

Hayat, T., Mustafa, M. and Pop, I. (2010): Heat and Mass Transfer for Soret and Dufour's Effect on Mixed Convection Boundary Layer Flow over a Stretching Vertical Surface in a Porous Medium Filled with a Viscoelastic Fluid, Communications in Nonlinear Science and Numerical Simulation, Vol. 15, pp. 1183-1196.

http://dx.doi.org/10.1016/j.cnsns.2009.05.062

Ibrahim, F. S., Hady, F.M., Abdel-Gaied, S.M. and Eid, M.R. (2010): Influence Of Chemical Reaction On Heat And Mass Transfer Of Non-Newtonian Fluid With Yield Stress By Free Convection From Vertical Surface In Porous Medium Considering Soret Effect, Applied Mathematics and Mechanics, Vol. 31, No. 6, pp. 675-684.

http://dx.doi.org/10.1007/s10483-010-1302-9

Jha, B. and Prasad, R. (1991): MHD Free-Convection And Mass Transfer Flow Through A Porous Medium With Heat Source, Astrophysics and Space Science, Vol. 181, No.1, pp.117-123. http://dx.doi.org/10.1007/BF00644118

Kafousias, N.G. and Raptis, A.A. (1981): Mass Transfer And Free Convection Effects On The Flow Past An Accelerated Vertical Infinite Plate With Variable Suction Or Injection, Review Romaine Science TechnologyMechanics Application, Vol. 26, pp.11-22.

Kairi, R.R. and Murthy, P.V.S.N. (2011): Effect Of Viscous Dissipation On Natural Convection Heat And Mass Transfer From Vertical Cone In A Non-Newtonian Fluid Saturated Non-Darcy Porous Medium, Applied Mathematics and Computation, Vol. 217, No. 20, pp. 8100-8114. http://dx.doi.org/10.1016/j.amc.2011.03.013

Kelly, D., Vajravelu, K. and Andrews, L. (1999): Analysis Of Heat And Mass Transfer Of A Viscoelastic, Electrically Conducting Fluid Past A Continuous Stretching Sheet, Nonlinear Analysis, Vol. 36, No. 6, pp. 767 784. http://dx.doi.org/10.1016/S0362-546X(98)00128-X

Kishore. P.M., Rajesh. V. and Varma. S. V.K. (2010): Effects Of Heat Transfer And Viscous Dissipation On MHD Free Convection Flow Past An Exponentially Accelerated Vertical Plate With Variable Temperature, Journal of Naval Architecture and Marine Engineering, Vol. 7, pp. 101-110. http://dx.doi.org/10.3329/jname.v7i2.4370

Narayana, M., Khidir, A.A., Sibanda, P. and Murthy, P.V.S.N. (2013): Soret Effect On The Natural Convection From A Vertical Plate In A Thermally Stratified Porous Medium Saturated With Non-Newtonian Liquid, Journal of Heat Transfer, Vol. 135, No. 3, art.no. 32501. http://dx.doi.org/10.1115/1.4007880

Pal, D. and Talukdar,B. (2010): Perturbation Analysis Of Unsteady Magneto Hydrodynamic Convective Heat And Mass Transfer In A Boundary Layer Slip Flow Past A Vertical Permeable Plate With Thermal Radiation And Chemical Reaction. Communications in Nonlinear Science and Numerical Simulation, Vol. 15, No. 7, pp. 1813-1830. http://dx.doi.org/10.1016/j.cnsns.2009.07.011

Pal, D. and Chatterjee,S. (2010): Heat And Mass Transfer In MHD Non-Darcian Flow Of A Micropolar Fluid Over A Stretching Sheet Embedded In A Porous Media With Non-Uniform Heat Source And Thermal Radiation, Communications in Nonlinear Science and Numerical Simulation, Vol. 15, No. 7, pp. 1843-1857. http://dx.doi.org/10.1016/j.cnsns.2009.07.024 
Rafael, C.B. (2007): Effects Of Heat Source/Sink, Radiation And Work Done By Deformation On Flow And Heat Transfer Of A Viscoelastic Fluid Over A Stretching Sheet. Computers and Mathematics with Applications, Vol. 53, pp. 305-316. http://dx.doi.org/10.1016/j.camwa.2006.02.041

Raju.M. C., AnandaReddy.N. and Varma, S.V.K. (2011): Hall-Current Effects On Unsteady MHD Flow Between Stretching Sheet And An Oscillating Porous Upper Parallel Plate With Constant Suction, Thermal science, Vol. 15, No. 2, pp. 45-48. http://dx.doi.org/org/10.2298/TSCI1102527C

Raju. M.C., Varma, S.V.K., Reddy P.V. and Sumon Saha. (2008): Soret Effects Due To Natural Convection Between Heated Inclined Plates With Magnetic Field, Journal of Mechanical Engineering, Vol. 39, pp. 43-48.

Reddy, N.A., Raju, M.C. and Varma, S.V.K. (2010): Unsteady Free Convective MHD Non Newtonian Flow Through A Porous Medium Bounded By An Infinite Inclined Porous Plate. International journal of Emerging Technologies and applications in engineering, Technology and sciences, Vol. 3, No. 2, 238-244.

Sivaraj, R. and Kumar, B.R. (2012): Unsteady MHD Dusty Viscoelastic Fluid Couette Flow In An Irregular Channel With Varying Mass Diffusion. International Journal of Heat and Mass Transfer, Vol. 55, pp. 30763089. http://dx.doi.org/10.1016/j.ijheatmasstransfer.2012.01.049

Tai, B.C. and Char, M. I. (2010): Soret And Dufour Effects On Free Convection Flow Of Non-Newtonian Fluids Along A Vertical Plate Embedded In A Porous Medium With Thermal Radiation, International Communications in Heat and Mass Transfer, Vol. 37, No. 5, pp. 480-483. http://dx.doi.org/10.1016/j.icheatmasstransfer.2009.12.017 